\title{
Phase Effects on the Conductance Through Parallel Double Dots
}

\author{
V.M. Apel ${ }^{1}$, Maria A. Davidovich ${ }^{1}$, G. Chiappe ${ }^{2}$ and E.V. Anda ${ }^{1}$ \\ ${ }^{1}$ Departamento de Física, Pontifícia Universidade Católica do Rio de Janeiro, 22453-900, Brazil \\ ${ }^{2}$ Departamento de Física, Facultad de Ciencias Exactas y Naturales, \\ Universidad de Buenos Aires, 1428, Argentina
}

\begin{abstract}
Phase effects on the conductance of a double-dot system in a ring structure threaded by a magnetic flux are studied. The Aharonov-Bohm effect combined with the dot many-body charging effects determine the phases of the currents going through each arm of the ring. The cases for zero magnetic flux or half a quantum of flux are discussed in detail. It is shown that, depending upon the magnetic flux and the state of charge of the dots, controlled by gate potentials, the dephasing of the upper and lower arm current gives rise to a $S=1 / 2$ or $S=1$ Kondo regime. We also show that even in the absence of a magnetic flux there can be a circulating current in the ring, depending on the system parameters.

PACS numbers: 73.63.-b,73.63.Kv
\end{abstract}

In the last years attention has been focused on the properties of double-quantum-dot systems $\frac{1}{-1}$ since they are expected to be basic building blocks for quantum computing ${ }^{2}$. In this context mesoscopic coherent transport is a key phenomenon that can probe entanglement ${ }^{3}$ and phases accumulated by electrons traversing the system. The phase-coherent transport effects can be analyzed by embedding the dots into an Aharonov-Bohm ring threaded by a magnetic flux and connected to leads. Theoretical $\underline{\underline{4}}^{\underline{4}}$ and experimental $1^{\underline{5}}$ works have discussed the transmission phase shifts of single and double quantum dot systems in the Coulomb blockade regime. More recently the effect of Kondo correlation on the transmission phase of

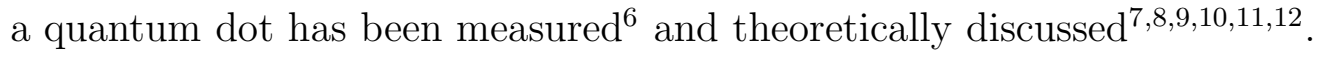

The two-quantum-dot system is particularly interesting since, when each dot is inserted into one arm of a ring connected to leads, as represented in Fig. 1, it presents two paths for the electrons to go through, producing interferences that depend upon the phase in each arm. The effect of the interferences on the spectral densities of such a system has been studied $\stackrel{14}{ }$, taking the intra and inter-dot Coulomb repulsion to be infinite. This limit restricts the study 
of the Kondo phenomenon and the phase shift interference effects to a situation in which the number of electrons in the system cannot be greater than one. However, depending on the magnetic flux enclosed by the ring, new and very interesting physics appear when both dots are charged and in the Kondo regime. Moreover, the general problem of the transmission phases in this system as the magnetic flux and the state of charge of the dots are varied has not been yet clarified. The dephasing between the two contributions to the current do not depend exclusively upon the state of charge of each dot, as a naive interpretation of the Friedel sum rule could predict. This is a consequence of the fact that the phases associated to the arms are not independent objects because one is renormalized by the other 7.8 .

The purpose of this letter is to contribute to the understanding of this problem. We discuss phase effects on the conductance of this system using an exact numerical diagonalization algorithm that provides only the ground state of the system. Our study is therefore restricted to zero temperature. This procedure permits us to take into account in an adequate way the interference effects due to the interaction between the two ring arms. Different Kondo regimes are accessed by varying the magnetic flux and the charge in the dots controlled by the gate potentials. The Aharonov-Bohm effect combined with the dot many-body charging effects determine the phases of the currents going through each arm of the ring. In order to have confiability in the numerical results, we adopt a dot-lead interaction such that the Kondo cloud is of the order of the cluster we exactly diagonalize $\underline{\underline{13}}$. In this case the plateau shape of the conductance, when the system is in the Kondo regime, is more like a Lorentzian. The conductance shows quite different behaviors for the magnetic flux $\Phi=0$ and $\Phi=\Phi_{0} / 2$. In the first case the dots act coherently and the system is in a Kondo state of total spin $S \sim 1$ while in the second, the dots are uncorrelated and in a $S=\frac{1}{2}$ Kondo regime. For other fluxes the conductance shows intermediate behavior between these two extremes. As an important consequence of interference we also find that current can circulate around the ring even in the absence of magnetic fluxes.

An Anderson two-impurity first-neighbor tight-binding Hamiltonian represents the system shown in Fig. 1,

$$
\begin{aligned}
H & =\sum_{\substack{r=\alpha, \beta \\
\sigma}}\left(V_{r}+\frac{U}{2} n_{r \bar{\sigma}}\right) n_{r \sigma}+t \sum_{i, j} c_{i \sigma}^{+} c_{j \sigma} \\
& +t^{\prime}\left[e^{i \frac{\pi}{2} \frac{\Phi}{\Phi_{0}}}\left(c_{\alpha \sigma}^{+} c_{1 \sigma}+c_{1 \sigma}^{+} c_{\beta \sigma}+c_{\beta \sigma}^{+} c_{\overline{1} \sigma}+c_{\overline{1} \sigma}^{+} c_{\alpha \sigma}\right)+\text { c.c. }\right]
\end{aligned}
$$


where $V_{\alpha}$ and $V_{\beta}$ are the gate potentials applied to the dots, $U$ is the Coulomb repulsion, considered to be equal for the two dots and $\Phi$ is the magnetic flux threading the ring. The parameters t' and t are, respectively, the hopping matrix element between the dots and their neighbors and between sites in the leads. The one particle Green functions $G$ are imposed to satisfy a Dyson equation $\hat{G}=\hat{g}+\hat{g} \hat{T} \hat{G}$ where $\hat{g}$ is the Green function matrix of a cluster containing the ring with the dots and a number of atoms of each lead and $\hat{T}$ is the matrix Hamiltonian that couples the cluster to the rest of the system. The undressed Green function $\hat{g}$ is calculated using the cluster ground state obtained by the Lanczos method. This approximation $\frac{13}{\underline{3}}$ has shown to be very accurate when the cluster is of the size of the Kondo cloud $h v_{F} / T_{K}$, where $v_{F}$ is the Fermi velocity and $T_{K}$ the Kondo temperature, although it gives qualitatively reliable results even for smaller clusters, compatible with the Friedel sum-rule and the Fermi liquid properties of the system.

The transport properties are studied as a function of the gate potentials applied to the dots. Taking the energies in units of the Coulomb interaction $U$, we set the leads bandwidth $W=8$ and $\Gamma=t^{2} / W=0.05$. The Fermi level is chosen to be $\epsilon_{F}=0$. The conductance $G=d I / d V$ is obtained using the Keldysh formalism to calculate the total current $I=$ $\sum_{i=\alpha, \beta} I_{i}$, where $I_{\alpha}$ and $I_{\beta}$ are the contributions coming from the two ring arms given by,

$$
I_{i}=\frac{2 e t^{\prime 2}}{h} \int_{-\infty}^{\infty}\left[\left(g_{L}^{-+}(\omega)-g_{R}^{-+}(\omega)\right) \times \operatorname{Im}\left\{G_{\overline{1} \overline{1}}^{r}(\omega) G_{\overline{1 i}}^{a}(\omega)-G_{\overline{1} 1}^{r}(\omega) G_{1 i}^{a}(\omega)\right\}\right] d \omega
$$

where sites 1 and $\overline{1}$ are the dots first neighbors, $G^{r}$ and $G^{a}$ are the dressed retarded and advanced Green functions and $g_{L}^{-+}$and $g_{R}^{-+}$are, respectively, the density of states at the left and right contacts multiplied by the Fermi distribution function. The Green functions are calculated at the Fermi level.

The conductance for two values of the magnetic flux, $\Phi=0$ and $\Phi_{0} / 2$, is represented in Fig. 2 as a function of the energy of the dot levels. It results to be weakly dependent upon the magnetic flux in the regions of the parameter space where only one dot is active so that current flows essentially along one arm of the ring. It possesses the characteristics of the one-dot conductance with a width, as a function of the gate potential, of the order of $U$ due to the Kondo effect of the charging dot. On the other hand, when $V_{\alpha} \sim V_{\beta}$, the conductance is strongly dependent upon the magnetic flux, showing special different features for the cases $\Phi=0$ and $\Phi_{0} / 2$. Let us focus on the region $-1<V_{\alpha}, V_{\beta}<0$ of Fig. 2. The differences in the conductance between these two cases are more striking when the dot level energies 
are similar, $\Delta E \sim 0$ (diagonal continuous line). In this case the two arms are at resonance since both dots are in the Kondo regime. For $\Phi=0$ the two arm currents are in phase and interfere constructively and the conductance has one broad peak. As the magnetic flux is turned on, the currents along the two arms are no longer in phase and the transport properties change qualitatively. For $\Phi=\Phi_{0} / 2$, the current arm amplitudes are out of phase and the conductance for $\Delta E=0$ cancels out for all values of the gate potentials, as shown in Fig. 2b.

For small values of $\Delta E$, the conductance presents two peaks for $\Phi=\Phi_{0} / 2$, and three for $\Phi=0$, the most interesting case. The spin-spin correlation, the charge at the dots and the conductance are displayed in Fig. 3 as a function of $V_{\alpha}$ for $\Delta E=0.6\left(V_{\alpha}=V_{\beta}-0.6\right)$, corresponding to the dashed line in Fig. 2. We first analyze the case $\Phi=0$. As $V_{\alpha}$ decreases from the value 0.5 and charge begins to enter into dot $\alpha$ its spin gets negatively correlated to the conduction electrons. The dot is in the Kondo regime and the conductance increases up to a quantum of conductance maximum around $V_{\alpha}=-0.2$. At $V_{\alpha}=-0.5$ the conductance cancels out and the various spin-spin correlations change abruptly. In the region $-0.5<V_{\alpha}<-1.1$ the Kondo correlation of both dots, $\left\langle\vec{S}_{\alpha} \vec{S}_{c}\right\rangle$ and $\left\langle\vec{S}_{\beta} \vec{S}_{c}\right\rangle$, gets stronger (Fig. 3b) and the conductance grows again reaching a second maximum at the electron-hole symmetry condition, $V_{\alpha}=-0.8$, where the system has just two electrons.

The most interesting aspect of the conductance is that its lateral peaks are due to a Kondo regime that is different from the one of the central peak. This conclusion can be obtained from the analysis of the spin-spin correlation of the two dots, shown in Fig. 3b. When the gate potential is reduced from $V_{\alpha}=0.5$, dot $\alpha$ enters into resonance and into the Kondo regime, while the other is well above the Fermi level and empty of charge. The current circulates only along dot $\alpha$ giving rise to the first peak of the conductance. It is a typical $S=1 / 2$ Kondo effect. As the gate potential is further reduced the electronic charge begins to drop also into dot $\beta$, giving rise to a new Kondo peak that, due to level repulsion, pushes the other one, at $\alpha$, to values below the Fermi level. This reduces the DOS at the Fermi level, diminishing the conductance of the system, and raises the dot $\alpha$ charge to a value above unity (Fig. 3c), increasing the energy of the ground state due to Coulomb interaction. As a consequence, a level crossing takes place and an excited state with total dots spin mean value $\left\langle S_{T}>\sim 1\right.$ and less charge at dot $\alpha$ becomes the new ground state of the system. This process gives rise to an abrupt reduction of the dot charge and to a restoration of 
the Kondo resonance at the Fermi level. While in the gate potential region corresponding to the original ground state the two dots are weakly antiferromagnetic correlated, in this new ground state they adopt a coherent behavior and have a strong ferromagnetic spin-spin correlation, $\left\langle\vec{S}_{\alpha} \vec{S}_{\beta}\right\rangle$, independent of the gate potential, as shown in Fig. 3b. The system is in a $S=1$ Kondo regime. The two crossing states have opposite parity. The crossing itself and the consequent abrupt change of the physical properties is derived from the fact that the system is spatially invariant under reflection so that parity is a good quantum number. When this symmetry is broken by introducing a small magnetic field (note that we are analyzing the case $\phi=0$ ) or an asymmetric perturbation to the Hamiltonian, the ground state results to be a linear combination of these two previous states. In this case the level crossing transforms into a cross-over behavior and the abrupt changes of the physical quantities are eliminated. This phenomenon is enhanced when the interaction among the dots and the conduction electrons is increased. When this interaction is large enough, the Kondo cloud is reduced to the size of the cluster we exactly diagonalize. In this region of the parameter space our approximation is almost numerically exact and independent of the cluster size we adopt, for sizes greater than the Kondo cloud.

The physics of the $\Phi=\Phi_{0} / 2$ case is diverse from the previously discussed. In this situation the dot spins are weakly correlated while each dot is independently Kondo correlated to the electrons of the leads, as appears in Fig. 3e. This different behavior can be clarified by a perturbation theory argument. The effective correlation between the dots can be obtained by taking the non-diagonal matrix elements that connect the dots to the rest of the circuit, $t^{\prime}$, as a perturbation. Due to the system topology it is clear that to get the dominant contribution to the effective inter-dot interaction it is necessary to go to forth order in perturbation theory. In this case, while for $\Phi=0$ the contributions that go from one dot to the other and return along the same path sum-up with the circulating contributions, for $\Phi=\Phi_{0} / 2$ these two contributions, having opposite signs, tend to cancel each other, giving rise to a weak interdot correlation. For small $\Delta E$ the conductance(Fig. 3d) possesses only one peak that develops into two as $\Delta E$ increases. The charge at the dots has a smooth dependence upon the gate potential, as shown in Fig. 3f.

In order to better understand these behaviors, we have calculated the conductance of each arm of the ring, using equation (2), as well as the phases of the electronic wave functions related to these two paths. The conductances are displayed in Fig. 4 as a function of the 
gate potential $V_{\alpha}$. For $\Delta E=0$ and $\Phi=0$ (Fig. 4a) the two arms are identical and their currents circulate along the ring in opposite direction summing up to constitute the total current in the leads. However, if $\Phi=\Phi_{0} / 2$ (Fig. 4b) the two arm currents circulate in the same direction giving rise to a persistent current along the ring and a total zero conductance. Satisfying a well known property, the circulating current changes sign as the total charge of the ring varies, as shown in the figure. The same phenomenon shows up in the case $\Phi=\Phi_{0} / 2$ and $\Delta E=0.6$, presented in Fig. $4 \mathrm{~d}$.

However, the existence of a magnetic flux crossing the ring is not a necessary condition to obtain a circulating current, as can be seen in Fig. 4c, for $\Phi=0$ and $\Delta E=0.6$. When the two dots are unequally charged there is a region of the gate potential parameter for which a net current circulates around the ring. This is due to an interference effect among the charges going along the two arms. For other values of the gate potentials, both currents are positive and the circulation disappears.

The quantum interference among the electrons are studied by calculating the phase and amplitude of the transmission that is proportional to the square modulus of $G_{1,1}^{r}=g_{c} t^{\prime}\left(G_{\alpha, 1}^{r}+\right.$ $\left.G_{\beta, 1}^{r}\right)$, where $g_{c}$ is the retarded Green function of the disconnected lead at the site $\overline{1}$ and the two terms represent the contributions coming from the two arms of the system. Taking the contribution from arm $\alpha$ as a reference, the total transmission can be written as $\mid A_{\alpha}+$ $\left.A_{\beta} e^{i \phi_{\alpha, \beta}}\right|^{2}$, where $A_{\alpha}$ and $A_{\beta}$ are, respectively, proportional to the modulus of $G_{\alpha, 1}^{r}$ and $G_{\beta, 1}^{r}$, and $\phi_{\alpha, \beta}$ is their phase difference. It is important to notice that $G_{\alpha, 1}^{r}$ and $G_{\beta, 1}^{r}$ incorporate all the renormalization of one arm due to the existence of the other. As a consequence, the phase of each trajectory is a function not only of its own dot charge but also of the charge of the other dot, and depends as well upon the topology of the whole system ${ }^{7}$. According to the Onsager relation the conductance of our system, that possesses a closed geometry, is an even function of the applied magnetic flux threading it, which implies $\phi_{\alpha, \beta}=0$ or $\pi$, depending upon the gate potentials applied to the dots. The transmission amplitudes and the phase difference are shown in Fig. 5 as a function of the gate potential for the case $\Phi=0, \Delta E=0.6$. When the transmission amplitudes are equal, $A_{\alpha}=A_{\beta}$, the conductance cancels or has a maximum depending upon the phase difference being $\phi_{\alpha, \beta}=\pi\left(V_{\alpha}=-0.5\right.$ and -1.1$)$ or $\phi_{\alpha, \beta}=0\left(V_{\alpha}=-0.8\right)$.

In summary we have studied a double-dot system in a ring threaded by a magnetic flux and connected to leads. The cases $\Phi=0$ and $\Phi_{0} / 2$ are discussed. It is shown that the 
dephasing of the upper and lower arm current determines whether the system is in a $S=1$ Kondo regime, when the two dots behave coherently, or in a $S=1 / 2$ Kondo regime, when they are uncorrelated. We show as well that, depending on the parameters, a circulating current appears even if there is no magnetic flux threading the ring. This very interesting phenomenon will be discussed in detail somewhere else.

We acknowledge FAPERJ, CNPq, CIAM (CNPq), CAPES(CAPG-BA,12/02) and CONICET for financial support. G.C. also acknowledges the support of the Buenos Aires University (UBACYT x210) and Fundacion Antorchas.

1 W.G.van der Wiel et al., Rev. Mod. Phys. 75, 1 (2003); C.A. Busser et al., Phys. Rev. B 62, 9907 (2000); D. Loss et al., Phys. Rev. A 57, 120 (1998).

2 D.P. DiVicenzo Science 720255 (1995); G. Burkard et al., Phys. Rev. B 592070 (1999).

3 D. Loss et al., Phys. Rev. Lett. 84, 1035 (2000).

4 A. Aharony et al., Phys. Rev. B 66, 115311 (2002); J. Konig et al., Phys. Rev. Lett. 86, 3855 (2001); A.Y. Smirnov et al., App. Phys. Lett. 77, 578 (2000).

5 A.W. Holleitner et al., Phys. Rev. Lett. 87, 256802 (2001); A. Yacoby et al., Phys. Rev. Lett. 74, 4047 (1995).

6 Y. Ji et al., Phys. Rev. Lett. 88, 076601 (2002); U. Gerland et al., Phys. Rev. Lett. 84, 3710 (2000).

7 A. Aharony et al., Phys. Rev. Lett. 90, 156802 (2003).

8 W. Hofstetter et al., Phys. Rev. Lett. 87, 156803 (2001).

9 E. Anda et al., Phys. Rev. B. 66, 035307 (2002).

10 K. Kang et al., Phys. Rev. B. 63, 113304 (2001)

11 H.P. Eckle et al., Phys. Rev. Lett. 87, 016602 (2001)

12 S.Y. Cho et al., Phys. Rev. B. 64, 033314 (2001)

13 V. Ferrari et al., Phys. Rev. Lett. 82, 5088 (1999).

14 D. Boese et al., Phys. Rev. B 66, 125315 (2002); ibid Phys. Rev. B 64, 125309 (2001). 


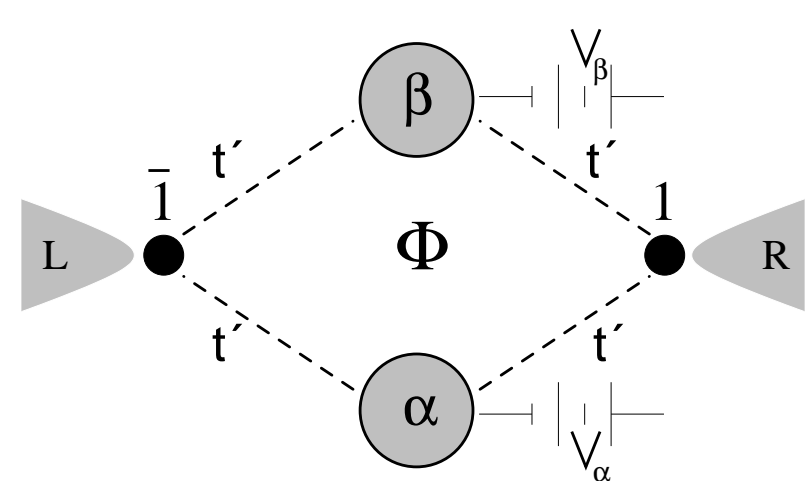

FIG. 1: Aharonov-Bohm interferometer with two embedded quantum dots, threaded by a magnetic flux $\Phi$, connected to leads.
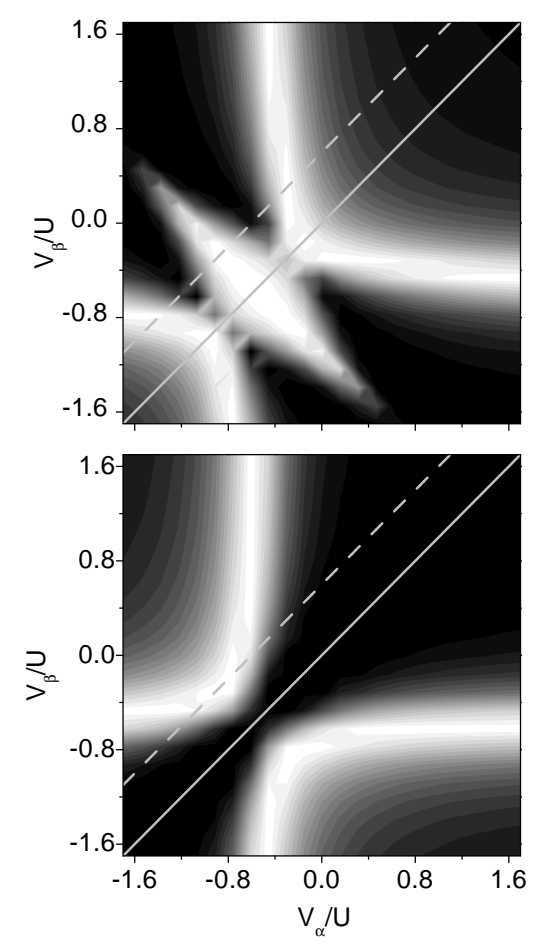

FIG. 2: Conductance (white, maximum; black, minimum) as a function of the gate potentials at the dots, $V_{\alpha}$ and $V_{\beta} . \Phi=0$ (upper panel), $\Phi=\Phi_{0} / 2$ (lower panel); $\Delta E=0$ (continuous line), $\Delta E=0.6$ (dashed line $)$. 


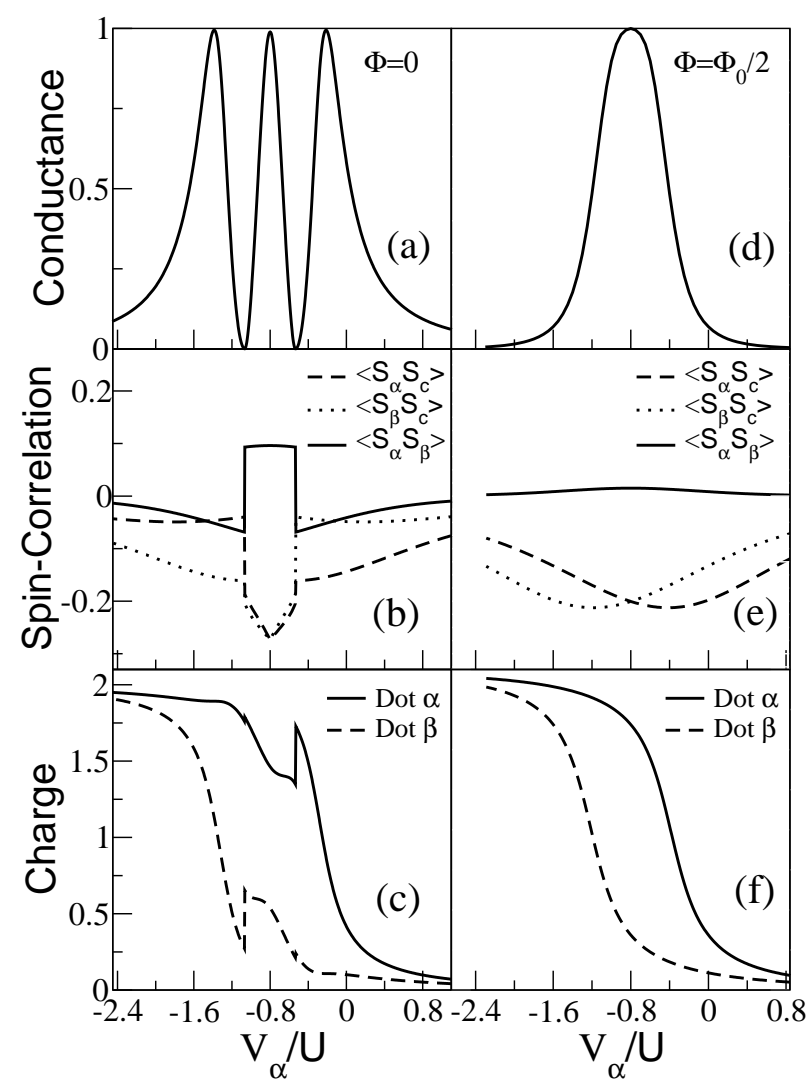

FIG. 3: Conductance (in units of $2 e^{2} / h$ ), spin correlation and charge (in units of e) at the dots for $\Delta E=0.6\left(V_{\beta}=V_{\alpha}+0.6\right)$ and $\Phi=0$ (left) and $\Phi=\Phi_{0} / 2$ (right), as a function of $V_{\alpha}$. 

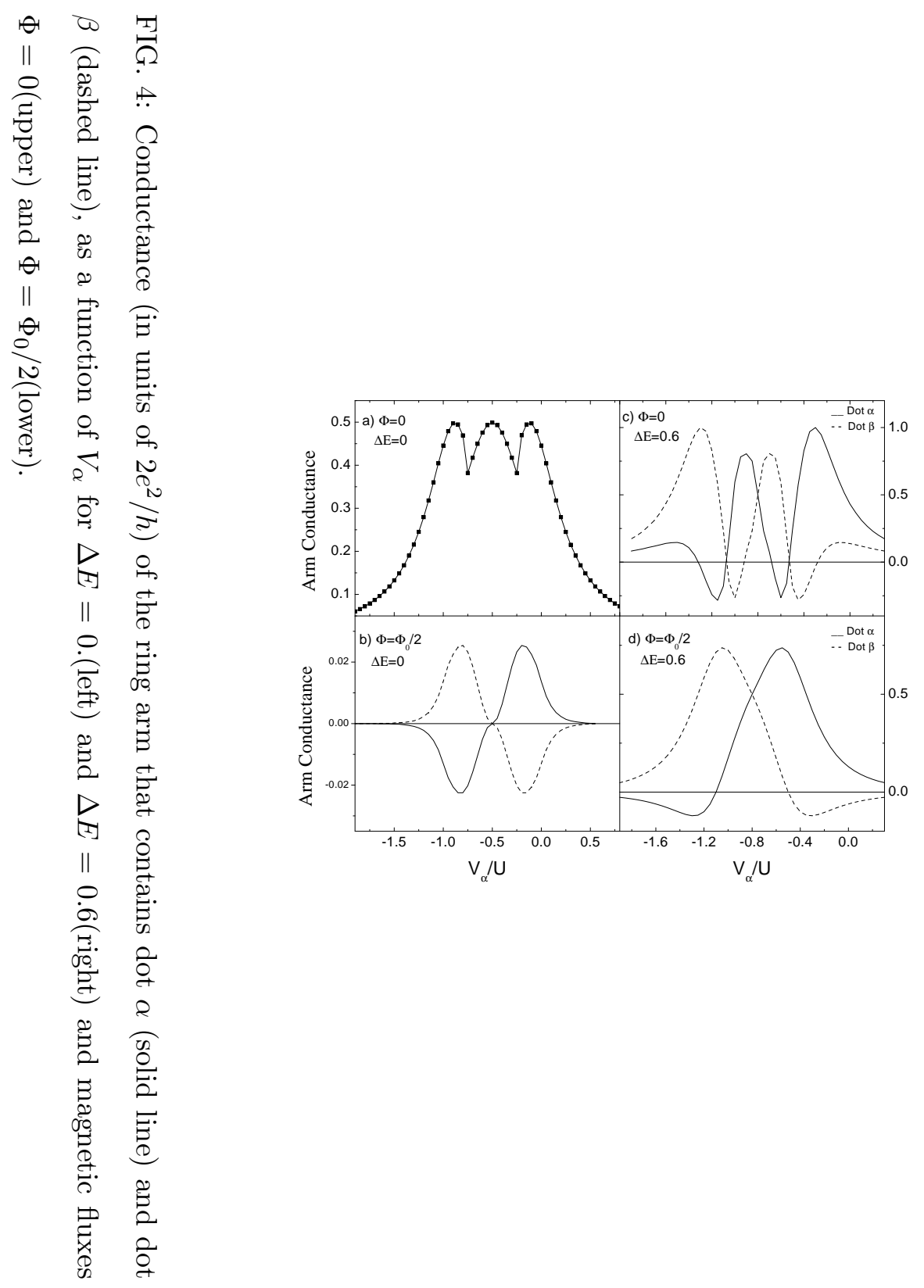


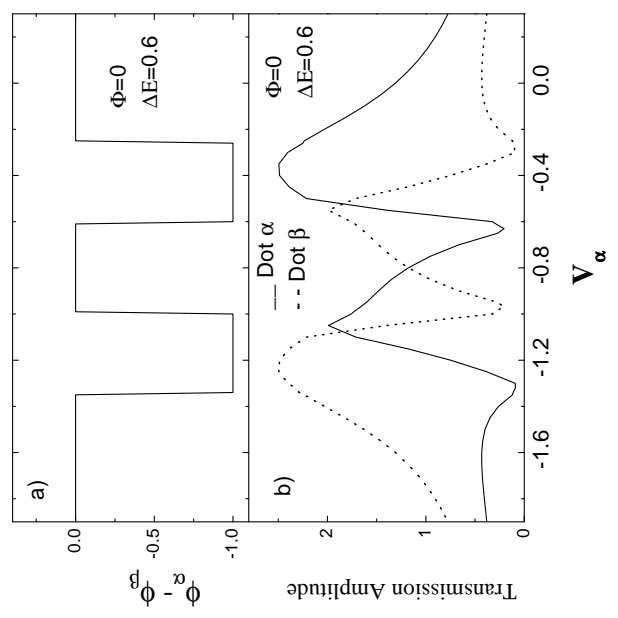

FIG. 5: Transmission through the two ring arms as a function of $V_{\alpha}$, for $\Phi=0$ and $\Delta E=0.6$. (a) phase difference (units of $\pi$ ); (b) modulus(arb.units). 